\title{
Clinical Characteristics and Outcome of Patients With Pheochromocytoma: A Single Center Tertiary Care Experience
}

\author{
Sara Sohail ${ }^{1}$, Waqas Shafiq ${ }^{1}$, Syed Abbas Raza ${ }^{1}$, Adnan Zahid ${ }^{1}$, Khurram Mir $^{2}$, Umal Azmat ${ }^{1}$ \\ 1. Endocrinology and Diabetes, Shaukat Khanum Memorial Cancer Hospital and Research Centre, Lahore, PAK 2. \\ Surgical Oncology, Shaukat Khanum Memorial Cancer Hospital and Research Centre, Lahore, PAK
}

Corresponding author: Sara Sohail,dr.sara.sohail@gmail.com

\section{Abstract \\ Objective}

The aim of this study was to evaluate the demographic and clinical characteristics of patients with pheochromocytoma and determine the treatment outcome with overall survival rates of patients with pheochromocytoma.

\section{Methods}

A retrospective, cross-sectional study was performed on all the patients with histologically proven pheochromocytoma presenting to Shaukat Khanum Memorial Cancer Hospital and Research Center (SKMCH \& RC) Lahore, between August 2006 and July 2018. Clinical, biochemical and radiological data were collected at presentation, post-surgery, at discharge and till the last follow-up; data was retrieved from hospital records. Cure was defined as no evidence of disease biochemically, clinically, and structurally.

\section{Results}

This study included 29 patients, $69 \%$ were female. The three most common symptoms were abdominal pain (51.7\%), hypertension (44.8\%) and headache (41.4\%). Most pheochromocytomas were sporadic (82.8\%), all were adrenal gland tumors, and $89.7 \%$ were unilateral. All patients underwent adrenalectomy. Open adrenalectomy was performed in 25 patients whereas four underwent laparoscopic adrenalectomy. Fifteen patients experienced postoperative complications. The most frequently documented intraoperative complication was hypotension. Death occurred in two patients, one patient died of metastatic disease secondary to malignant pheochromocytoma and the other patient died from perioperative complications. Cure was documented biochemically and/or radiologically in $96.5 \%$ patients.

\section{Conclusions}

Received 04/05/2020 Review began 04/15/2020 Review ended 04/27/2020 Published 05/06/2020

(๑) Copyright 2020

Sohail et al. This is an open access article distributed under the terms of the Creative Commons Attribution License CC-BY 4.0., which permits unrestricted use, distribution, and reproduction in any medium, provided the original author and source are credited.
Abdominal pain was predominant presenting feature most likely due to large tumor size. Most patients presenting to SKMCH \& RC, had large intra-abdominal tumors with high cure rate. Mortality was low despite high rate of perioperative complications.

Categories: Endocrinology/Diabetes/Metabolism, Internal Medicine, Urology

Keywords: plasma metanephrine and normetanephrine levels, adrenal medulla, pheochromocytoma, catecholamines, urinary vanillylmandelic acid (24-hour urinary vma)

\section{Introduction}

Pheochromocytoma is a tumor arising from adrenomedullary chromaffin cells that commonly produces one or more catecholamines [1]. About $80 \%$ to $85 \%$ of all chromaffin-cell tumors are pheochromocytomas [2]. The incidence of pheochromocytoma is two to eight per million persons per year [3]. It is found in $0.1 \%$ to $1 \%$ of patients presenting with hypertension and as Young et al. reported approximately $5 \%$ of patients with incidentally discovered adrenal masses [4-6,7]. The peak incidence occurs in the third to fifth decade of life. The mean age at diagnosis is 24.9 years in hereditary cases and 43.9 years in sporadic cases [8]. It occurs more often in females than in males [9]. Most catecholamine secreting tumors are sporadic. However, about $25 \%$ of patients have the disease as part of a hereditary syndrome [8-10]. The inherited familial syndromes associated with pheochromocytoma include multiple endocrine syndrome type 2 (MEN 2), von HippelLindau (VHL) syndrome and neurofibromatosis type 1 (NF-1) $[2,11]$. Pheochromocytoma has malignant potential as well. Malignancy is defined as the presence of metastases in non-chromaffin tissue. The prevalence of malignancy varies between $10 \%$ and $17 \%$ [12].

The classic triad of clinical features consists of episodic headaches, palpitations, and diaphoresis [13]. The hallmark clinical finding is hypertension, which can be paroxysmal or sustained. Hypertensive crisis can lead to cardiac arrhythmias, myocardial infarction, and even death. When the tumor is suspected clinically, 
biochemical confirmation, consisting of plasma and urinary catecholamine (adrenaline, noradrenaline) and their metabolites (metanephrine and normetanephrine) is required. This should be followed by tumor localisation using various imaging studies $[2,11]$. Computed tomography (CT) imaging or magnetic resonance imaging (MRI) of the abdomen and pelvis (at least through the level of the aortic bifurcation) are the most commonly used methods and have similar sensitivities (90\%-100\%) and specificities (70\%-80\%) for tumor localisation [14]. Surgical resection followed by postoperative monitoring is the safest and the most effective therapeutic intervention for pheochromocytoma [15].

Data regarding survival of patients with localized (benign) disease or regional disease is not clearly available. Patients with localized disease are expected to experience an overall survival approaching that of age matched disease-free individuals but $6.5 \%$ to $16.5 \%$ of these patients will develop a recurrence, usually 5 to 15 years after initial surgery and approximately $50 \%$ of the patients with recurrent disease experience distant metastasis $[16,17]$. The five-year survival in the setting of metastatic disease (whether identified at the time of initial diagnosis or identified post operatively as recurrent disease) is $40 \%$ to $45 \%$ [18]. Longterm follow-up is essential for all patients with pheochromocytoma even when initial pathology demonstrates no findings that are concerning for malignancy. After resection of a solitary sporadic pheochromocytoma, patients should undergo baseline postoperative biochemical testing followed by annual lifelong biochemical testing. Patients with hereditary syndromes of pheochromocytoma who have undergone resection, in addition to lifelong annual biochemical screening of pheochromocytoma, routine screening for other component tumors of their specific syndrome is indicated [6].

There are several reports and data available on pheochromocytoma from centres in developed countries, but there is limited information available from developing regions of the world [19,20]. This can be related to limited healthcare facilities, unavailability of specific tests for diagnosis of pheochromocytoma. Single institution experience is also limited in our part of world with paucity of local data. Due to rarity of this tumor, to date there is no study conducted on pheochromocytoma patients, on their various presentation and outcomes from Pakistan. The aims of this study were to look into the demographics, different clinical presentation of pheochromocytoma, to determine the treatment outcome of these patients. This study will further help in recognition of symptoms and early referral leading to proper management of this tumor.

\section{Materials And Methods}

A retrospective cross-sectional study was performed on all the patients with pheochromocytoma presenting to Shaukat Khanum Memorial Cancer Hospital and Research Centre (SKMCH \& RC) Lahore, between August 2006 and July 2018. The study was approved by Institutional Review Board committee of SKMCH \& RC (EXMPT-13-08-18-01).

Clinical information on all patients with histologically proven pheochromocytoma was retrieved from hospital records. For each patient, information at presentation, post-surgery, at discharge and till the last follow-up was recorded by the primary author and noted in a predefined questionnaire. No patients were excluded from the study over the study period.

Laboratory tests were done at SKMCH \& RC. Pre- and post-surgery hormonal assessment for pheochromocytoma was done for all patients and was retrieved from hospital records and noted in questionnaire. Until 2015, 24-hour urine vanillylmandelic acid (VMA) test was performed. After 2015, plasma metanephrine and normetanephrines levels were standard hormone assessment for these patients.

Radiological investigations were performed which included computed tomography scan (CT scan), magnetic resonance imaging (MRI) and Iodine I 123-metaiodobenzylguanidine (MIBG) scintigraphy. Tumor size was recorded on histology samples and was retrieved from patient's hospital record. Pheochromocytoma of adrenal gland scoring scale (PASS Score) based on different histological parameters, was calculated [21].

Postoperative patients were followed biochemically and radiologically at regular interval. Biochemical cure was defined as normal plasma metanephrine and normetanephrine levels post-operatively. Structural cure was determined by absence of tumor on radiological imaging post-surgery. For the outcomes, inpatient and outpatient notes, presurgery and post-surgery biochemical results, and scans of patients' follow-up at hospital after their surgical procedures were being reviewed.

Furthermore, age, sex, body mass index, tumor characteristics (tumor stage, grade, histology) were treated as independent variables. Outcome response (alive or death) was treated as dependent variable.

Statistical Package for the Social Sciences (SPSS) version 20 (IBM Corp., Armonk, NY) was used for statistical analysis. Descriptive statistics were used and categorical data was analysed by calculating frequencies, percentages (gender). Continuous variables were reported in terms of mean and standard deviation (age).

\section{Results}

A total of 29 patients (20 females) were included in the study. Mean age at presentation was $36.2+12.5$ 


\section{Cureus}

years. The majority of patients were from Punjab (55.2 \%) followed by Khyber Pakhtunkha (KPK) (31.0\%) and Afghanistan (13.8\%). The rest of the demographic data and baseline characteristics are given in Table 1.

\begin{tabular}{|l|l|}
\hline Characteristic & N (\%) \\
\hline Age & $36.24+12.49$ years \\
Mean & 20 to 64 years \\
Range & \\
Gender & $9(31 \%)$ \\
Male & $20(69 \%)$ \\
Female & \\
BMI (kg/m $\left.{ }^{2}\right)$ & $25.77+14.59$ \\
Mean & 18.29 to 31.64 \\
Range & \\
Co-morbidity at Presentation & $5(17.2 \%)$ \\
Diabetes & $3(10.3 \%)$ \\
\hline Hypertension & \\
\hline
\end{tabular}

TABLE 1: Baseline characteristics of patients with pheochromocytoma at presentation $(n=29)$

BMI: Body Mass Index

The three most common clinical presentations were abdominal pain (51.7\%), hypertension (44.8\%) and headache (41.1\%), whereas pheochromocytoma was an incidental finding in four patients (13.8\%). The classical triad of headache, palpitation and sweating was seen in only three patients (10.3\%). At presentation, mean heart rate was 87.7 beats per min (62-130), mean systolic blood pressure was $140.9 \mathrm{~mm}$ $\mathrm{Hg}$ (110-210) and mean diastolic blood pressure was $89.15 \mathrm{~mm} \mathrm{Hg}$ (60-150). Two of our cases presented with hypertensive crisis, one with vision loss secondary to malignant hypertension and the other developed hypertensive crisis during and invasive procedure. The various different clinical features at presentation in patients with pheochromocytoma are detailed in Table 2. 


\section{Cureus}

Clinical Presentation

Abdominal Pain

Hypertension

Headache

Palpitation

Sweating

Abdominal Mass

Incidental

Classical Triad

Tumor Laterality

Right sided

Left sided

Bilateral

Tumor Size (cm)

Mean

Range

PASS Score

PASS Score $<4$

PASS score $>4$

PASS Score not available

\section{Total Percentage}

$15(51.7 \%)$

$13(44.8 \%)$

$12(41.1 \%)$

$10(34.5 \%)$

$5(17.2 \%)$

5 (17.2\%)

4 (13.8\%)

3 (10.3\%)

$14(48.3 \%)$

$12(41.4 \%)$

$3(10.3 \%)$

$8.31+3.5$

4 to $18 \mathrm{~cm}$

$20(69 \%)$

$6(20.7 \%)$

3 (10.3\%)

TABLE 2: Clinical features at presentation in patients with pheochromocytoma $(n=29)$

PASS Score: Pheochromocytoma of adrenal gland scoring scale

All patients presented with intra-abdominal tumors that originated from adrenal gland. Only three (10.3\%) of these patients had bilateral pheochromocytoma.

The majority (82.8\%) of patients had sporadic tumors. Five (17.2\%) patients had familial syndromes MEN type $2 \mathrm{a}$ based on the presence of medullary thyroid cancer and pheochromocytoma concomitantly. Pheochromocytoma was bilateral in three (60\%) and unilateral in two (40\%) of these patients.

Plasma metanephrine and normetanephrine levels were checked in 19 patients (65.5\%), 24-hour urinary VMA levels were checked in six patients (20.7\%). Hormonal assessment was not available in four patients (13.8\%). Twelve patients (63.2\%) had increased plasma metanephrine and normetanephrine levels and seven patients (36.8\%) had increased normetanephrine levels only. Twenty-four hour urinary VMA levels were normal in all six patients (20.7\%).

At presentation, all patients had CT scan for initial tumor localisation. Eleven patients had MRI scans; only one patient showed evidence of metastasis. In 26 patients, CT or MRI scans correctly identified tumor site before surgery. In two patients, tumor was initially diagnosed as pancreatic tail mass and in one patient tumor was initially diagnosed as extra adrenal which later, on further imaging studies, revealed an adrenal mass. Baseline MIBG scans were done in 20 patients; of these all correctly identified the tumor preoperatively.

All patients underwent adrenalectomy. Open adrenalectomy was performed in 25 patients whereas four patients underwent laparoscopic adrenalectomy. Perioperative complications are presented in Table 3. 


\section{Cureus}

\begin{tabular}{|l|l|l|}
\hline Complications & Preoperative Alpha and Beta blockade $(\mathbf{n}=\mathbf{2 0})$ & No medical management preoperative $(\mathbf{n}=\mathbf{7})$ \\
\hline Perioperative Complications & 10 & 5 \\
Hypotension & 9 & 2 \\
Haemorrhage & 1 & 1 \\
Hypertensive crisis & none & 2 \\
\hline
\end{tabular}

TABLE 3: Therapeutic modalities and perioperative complications in patients with pheochromocytoma $(n=27)$

Twenty patients received alpha and beta blockade prior to surgery. Postoperative complications were reported in 15 patients and they were more common in patients who did not receive preoperative alpha and beta blockade. There was no death reported in perioperative period.

On histopathology mean tumor size was $8.3 \mathrm{~cm}(5 \mathrm{~cm}$ to $18 \mathrm{~cm})$. PASS Score was calculated in 26 patients. Twenty patients were noted to have PASS score less than 4 and the rest of six patients had PASS score greater than equal to 4 . Immunohistochemical staining (IHC) was performed in 27 patients, details are listed in Table 4.

Immunohistochemical staining
Chromogranin
Synaptophysin
Synaptophysin and Chromogranin
Synaptophysin and S-100
Chromogranin and S-100

Number of patients
9
2
6
1
4
5

TABLE 4: Immunohistochemical staining pattern in pheochromocytoma patients

Complete biochemical and structural cure post surgery was achieved in 28 patients. Normalization of blood pressure was achieved in all pheochromocytoma patients who presented with hypertension as initial clinical finding. Mean patient follow-up was 16.5 months (two months to 58 months). None of these patients showed evidence of disease recurrence except one patient who presented with metastatic disease. Two mortalities were recorded, one patient died due to metastatic pheochromocytoma, the second patient died of adrenal crisis as she had undergone bilateral adrenalectomy for bilateral pheochromocytoma.

\section{Discussion}

Our study has shown that peak incidence of pheochromocytoma occurs in the fourth to fifth decades of life and is more common in female. There was no extra adrenal pheochromocytoma in our study. On average, every sixth patient had pheochromocytoma as a part of a hereditary syndrome (MEN-2A). These results were lower than that reported by Safwat et al. $(\mathrm{n}=17,14.9 \%)[22]$.

The most frequent clinical presentation was abdominal pain (likely secondary to mass effect) followed by hypertension. The classical triad (headache, palpitations, sweating) was only present in minority of patients. Similar presentations have also been reported in other studies as well [22,23]. These studies have reported a higher rate of hypertension than we observed. One possible reason for this is that about one-sixth of our patients had medullary thyroid cancer (MTC) and only on further investigations were found to have pheochromocytoma and did not initially present to us for evaluation of an adrenal lesion. Hypertension was observed in only one patient with hereditary syndrome and similarly hypertension was not observed in any pheochromocytoma patients who were diagnosed incidentally. Genetic testing is not available in our center; hence all patients who were diagnosed with MTC underwent further biochemical and cross-sectional imaging to rule out pheochromocytoma and primary hyperparathyroidism. 
Prior to 2015, 24-hour urinary VMA was the screening test for pheochromocytoma being used at our center. All six patients had normal urinary VMA level but on histopathology were proven to be pheochromocytoma. These results are in accordance with low sensitivity of this test as reported by Lenders et al. in 2002 [24]. Plasma metanephrine and normetanephrine levels were checked in 19 patients and were raised in all of them. This adheres to international literature in which specificity and sensitivity of this test has been reported as 98 and 100\% [25]. This study also proved that when compared between 24 hour urinary VMA and plasma metanephrines, the later provide the best test for excluding or confirming pheochromocytoma and should be the preferred test for diagnosis of the tumor among the two. Cross-sectional CT/MRI was able to correctly localize all tumors preoperatively, similar to other studies [19,22]. MIBG correctly identified the tumor in all cases, with MIBG sensitivity being $100 \%(n=20)$; this is slightly higher than that reported by Berglund et al. and Shapiro study which was $88-98 \%$, and may be related to large tumor sizes [26,27].

An open adrenalectomy was performed in majority of our patients (86.2\%), most likely in view of large tumor size identified on preoperative imaging. Perioperative complications were present in about $50 \%$ of our patient which is consistent with literature but is slightly higher than that reported by Kinney et al. [28]. This relatively higher complication rate in our study is likely due to absence of alpha and beta blockade in some patients per operatively as has been stated in results.

Tumor size in our study (mean $8.3 \mathrm{~cm}$ ) was larger than that recorded in other studies like Lo et al. and Zorgani et al., in both studies mean tumor size reported $6.4 \mathrm{~cm}[19,29]$. This may be accounted for by late presentation or delayed referral for specialized care. Biochemical, structural and clinical cure was achieved in $96.4 \%$ of our patients. This cure rate is higher (70-92.4\%) than that reported in other studies $[2,19,23]$. The reason for this higher cure rate can be as there was only one patient with malignant pheochromocytoma in our study and hence with metastatic disease, secondly follow-up period in our patients (mean follow-up 16.5 months) was less than that in other studies. Overall mortality rate in pheochromocytoma is low and various studies like Zorgani et al., Huddle, have reported it to be 5.9-7.4\% [23,29]. Our study also showed a low mortality rate of $6.9 \%$. The reason for low mortality is due to adequate alpha and beta blockade in perioperative period, in our study patients did experience higher perioperative complications rate but those were adequately managed and overall mortality was low and none from perioperative complications.

This study has several limitations. A retrospective study was performed but data was extracted using electronic hospital software and collaborated with patient visits to other clinics within the hospital. This would reduce recall any selection bias which traditionally makes retrospective studies weak, standardized biochemical testing was not performed for all pheochromocytoma patients, it was a single center study, and although pheochromocytoma is a rare diagnosis the sample size of patients was small. However, regardless of small number, this is a novel study as there is scarcity of data available regarding clinical presentation and outcome of pheochromocytoma in this part of subcontinent.

\section{Conclusions}

This study highlights the importance of keeping high index of suspicion for pheochromocytoma at primary health care levels which would lead to early referrals. Furthermore, pheochromocytoma can have varying presentations and hypertension is not necessarily a predominant presentation. More studies are required specially from this part of subcontinent to further confirm the results of this study.

\section{Additional Information \\ Disclosures}

Human subjects: Consent was obtained by all participants in this study. Institutional Review Board (IRB) of Shaukat Khanum Memorial Cancer Hospital and Research Centre issued approval EXMPT-13-08-18-01. Institutional Review Board (IRB) of Shaukat Khanum Memorial Cancer Hospital and Research Centre, Lahore, Pakistan issued approval of the study protocol EXMPT-13-08-18-01. IRB considers the fact that all information to be collected in this study is already existing in medical records. IRB further considers that this information will be recorded on data extraction sheet in such a manner that subjects cannot be identified directly or indirectly through use of codes. IRB also grants this study waiver of informed consent. On the basis of all above, IRB grants this study an exempt status. Animal subjects: All authors have confirmed that this study did not involve animal subjects or tissue. Conflicts of interest: In compliance with the ICMJE uniform disclosure form, all authors declare the following: Payment/services info: All authors have declared that no financial support was received from any organization for the submitted work. Financial relationships: All authors have declared that they have no financial relationships at present or within the previous three years with any organizations that might have an interest in the submitted work. Other relationships: All authors have declared that there are no other relationships or activities that could appear to have influenced the submitted work.

\section{References}

1. Erickson D, Kudva YC, Ebersold MJ, Thompson GB, Grant CS, Heerden JV, Young WF Jr: Benign paragangliomas: clinical presentation and treatment outcomes in 236 patients. J Clin Endocrinol Metab. 2001, 86:5210-5216. 10.1210/jcem.86.11.8034 
2. Lenders JWM, Eisenhofer G, Mannelli M, Pacak K: Phaeochromocytoma. Lancet. 2005, 366:665-675. 10.1016/S0140-6736(05)67139-5

3. Stenstrom G, Svardsudd K: Pheochromocytoma in Sweden 1958-1981: an analysis of the National Cancer Registry Data. Acta Med Scand. 1986, 220:225-232. 10.1111/j.0954-6820.1986.tb02755.x

4. Sinclair AM, Isles CG, Brown I, Cameron H, Murray GD, Robertson JW: Secondary hypertension in a blood pressure clinic. Arch Intern Med. 1987, 147:1289-1293. 10.1001/archinte.1987.00370070103015

5. Anderson GH Jr, Blakeman N, Streeten DH: The effect of age on prevalence of secondary forms of hypertension in 4429 consecutively referred patients. J Hypertens. 1994, 12:609-615. 10.1097/00004872199405000-00015

6. Omura M, Saito J, Yamaguchi K, Kakuta Y, Nishikawa T: Prospective study on the prevalence of secondary hypertension among hypertensive patients visiting a general outpatient clinic in Japan. Hypertens Res. 2004, 27:193-202. 10.1291/hypres.27.193

7. Young WF Jr: Management approaches to adrenal incidentalomas: a view from Rochester, Minnesota . Endocrinol Metab Clin North Am. 2000, 29:159-185. 10.1016/s0889-8529(05)70122-5

8. Neumann HP, Bausch B, McWhinney SR, et al.: Germ-line mutations in nonsyndromic pheochromocytoma. N Engl J Med. 2002, 346:1459-1466. 10.1056/NEJMoa020152

9. Klingler HC, Klingler PJ, Martin JK Jr, Smallridge RC, Smith SL, Hinder RA: Pheochromocytoma. Urology. 2001, 57:1025-1032. 10.1016/s0090-4295(01)00966-9

10. Jiménez C, Cote G, Arnold A, Gagel RF: Should patients with apparently sporadic pheochromocytomas or paragangliomas be screened for hereditary syndromes?. J Clin Endocrinol Metab. 2006, 91:2851-2858. 10.1210/jc.2005-2178

11. Young WF Jr: Adrenal causes of hypertension: pheochromocytoma and primary aldosteronism . Rev Endocr Metab Disord. 2007, 8:309-320. 10.1007/s11154-007-9055-Z

12. Lenders JW, Duh QY, Eisenhofer G, et al.: Pheochromocytoma and paraganglioma: an endocrine society clinical practice guideline. J Clin Endocrinol Metab. 2014, 99:1915-1942. 10.1210/jc.2014-1498

13. Bravo EL, Gifford RW Jr: Pheochromocytoma: diagnosis, localization and management. N Engl J Med. 1984, 311:1298-1303. 10.1056/NEJM198411153112007

14. Ilias I, Pacak K: Current approaches and recommended algorithm for the diagnostic localization of pheochromocytoma. J Clin Endocrinol Metab. 2004, 89:479-491. 10.1210/jc.2003-031091

15. Plouin PF, Duclos JM, Soppelsa F, Boublil G, Chatellier G: Factors associated with perioperative morbidity and mortality in patients with pheochromocytoma: analysis of 165 operations at a single center. J Clin Endocrinol Metab. 2001, 86:1480-1486. 10.1210/jcem.86.4.7392

16. Plouin PF, Chatellier G, Fofol I, Corvol P: Tumor recurrence and hypertension persistence after successful pheochromocytoma operation. Hypertension. 1997, 29:1133-1139. 10.1161/01.hyp.29.5.1133

17. van Heerden JA, Roland CF, Carney JA, Sheps SG, Grant CS: Long-term evaluation following resection of apparently benign pheochromocytoma(s)/paraganglioma(s). World J Surg. 1990, 14:325-329. $10.1007 /$ bf01658516

18. Averbuch SD, Steakley CS, Young RC, Gelmann EP, Goldstein DS, Stull R, Keiser HR: Malignant pheochromocytoma: effective treatment with a combination of cyclophosphamide, vincristine, and dacarbazine. Ann Intern Med. 1988, 109:267-273. 10.7326/0003-4819-109-4-267

19. Lo CY, Lam KY, Wat MS, Lam KS: Adrenal pheochromocytoma remains a frequently overlooked diagnosis . Am J Surg. 2000, 179:212-215. 10.1016/s0002-9610(00)00296-8

20. Modigliani E, Vasen HM, Raue K, et al.: Pheochromocytoma in multiple endocrine neoplasia type 2: European study. J Intern Med. 1995, 238:363-367. 10.1111/j.1365-2796.1995.tb01211.x

21. Thompson LD: Pheochromocytoma of the adrenal gland scaled score (PASS) to separate benign from malignant neoplasms: a clinicopathologic and immunophenotypic study of 100 cases. Am J Surg Pathol. 2002, 26:551-566. 10.1097/00000478-200205000-00002

22. Safwat AS, Bissada NK, Seyam RM, Al Sobhi S, Hanash KA: The clinical spectrum of phaeochromocytoma: analysis of 115 patients. BJU Int. 2008, 101:1561-1564. 10.1111/j.1464-410X.2008.07430.x

23. Huddle KRL: Phaeochromocytoma in black South Africans: a 30-year audit. S Afr Med J. 2011, 101:184-188. 10.7196/SAMJ.4320

24. Lenders JW, Pacak K, Walther MM, et al.: Biochemical diagnosis of pheochromocytoma: which test is best? JAMA. 2002, 287:1427-1434. 10.1001/jama.287.11.1427

25. Hickman PE, Leong M, Chang J, Wilson SR, McWhinney B: Plasma free metanephrines are superior to urine and plasma catecholamines and urine catecholamine metabolites for the investigation of phaeochromocytoma. Pathology. 2009, 41:173-177. 10.1080/00313020802579284

26. Berglund AS, Hulthen UL, Manhem P, Thorsson O, Wollmer P, Törnquist C: Metaiodobenzylguanidine (MIBG) scintigraphy and computed tomography (CT) in clinical practice. Primary and secondary evaluation for localization of phaeochromocytomas. J Intern Med. 2001, 249:247-251. 10.1046/j.13652796.2001.00792.x

27. Shapiro B: Imaging of catecholamine-secretion tumours: uses of MIBG in diagnosis and treatment . Baillieres Clin Endocrinol Metab. 1993, 7:491-507. 10.1016/s0950-351x(05)80185-5

28. Kinney MA, Warner ME, vanHeerden JA, et al.: Perianesthetic risks and outcomes of pheochromocytoma and paraganglioma resection. Anesth Analg. 2000, 91:1118-1123. 10.1097/00000539-200011000-00013

29. Zorgani AE, Pirie FJ, Motala AA: Characteristics and outcome of patients with pheochromocytoma at a tertiary endocrinology clinic in Durban, South Africa over 14 years. J Endocrinol Metab Diabetes South Africa. 2018, 23:52-58. 10.1080/16089677.2018.1446592 